\title{
Wie weiter in der ambulanten Medizin?
}

Einmal mehr konnte sich das allgemeine Interesse im Parlament nicht durchsetzen, was ausserordentlich schade ist!

Nach dem Nein des Ständerates hatten die FMH und die Konferenz der kantonalen Gesundheitsdirektoren (GDK) ihren Vorschlag zur Steuerung des Gesundheitssystems reaktiviert. Dieser beruht auf den aktuellen Bedürfnissen und ist auf eine Nachfolge- oder Übergangsregelung des Zulassungsstopps ausgerichtet.

Doch nein, man hat nun einfach noch einen draufgesetzt und den Zulassungsstopp um zwei Jahre verlängert und die Lösung nicht berücksichtigt, die damals auf Wunsch des Parlaments von den «betroffenen Kreisen» erarbeitet wurde - jene Kreise, die immerhin die Abstimmung vom letzten Juni gewonnen haben! Unsere konstruktive, offene und parteiunabhängige Lösung hatte schliesslich auch den Vorsteher des Eidgenössischen Departements des Innern überzeugt.

Die erneute Verlängerung des Zulassungsstopps ist keine Lösung, da bis zum Anfang 2012 trotzdem neue Instrumente für die Steuerung des Gesundheitswesens vorliegen müssen.

\section{Wir fordern das Parlament auf, die medizinischen Fachgebiete, für die der Zulassungsstopp künftig nicht mehr gilt, breit und klar zu definieren. Da kein Steuerungsinstrument zur Verfügung steht, ist eine solche Definition für die medizinische Versorgung unseres Landes von grösster Bedeutung}

Die in der Grundversorgung tätigen Ärzte sind von der Verlängerung des Zulassungsstopps zwar ausgenommen, doch im Gegensatz zum Vorschlag der FMH/GDK sind keine Unterstützungsmassnahmen für die Hausärzte vorgesehen, obwohl diese eine Unterstützung dringend benötigen.

In der Praxis zielt diese Pseudolösung an den gegenwärtigen Problemen unseres Gesundheitswesens vorbei. Ob das wohl Absicht ist? Möchten vielleicht gewisse Kreise ein Chaos anrichten, damit sie anschliessend notfallmässig Massnahmen - wie etwa die Vertragsfreiheit - anordnen können, von denen sie schon lange träumen? Hoffen sie oder andere Kreise darauf, dass die Marktwirtschaft in zwei Jahren wieder en vogue ist und sie endlich den wirtschaftlichen Wettbewerb auch in den Behandlungszimmern einführen können?

Überlassen wir jedoch die Mutmassungen anderen und wenden wir uns den Problemen zu, mit denen uns die neue Ausgangslage konfrontiert.
Der «Antrag Triponez» lässt jene medizinischen Fachbereiche im Stich, die unterstützt werden sollten, um die medizinische Versorgung der Bevölkerung zu gewährleisten: Hausarztmedizin, Psychiatrie und Pädiatrie, um nur die Fachbereiche zu nennen, die am stärksten gefährdet sind.

Wenn kein Nachwuchs vorhanden ist, nützt es herzlich wenig, die Schleusen für die Grundversorger zu öffnen; die Kantone müssen deshalb dringend Steuerungsmöglichkeiten haben, damit sie Anreize schaffen und die medizinische Versorgung in peripheren Gebieten sicherstellen können.

Die Definition des Begriffs «Grundversorger» ist keine leichte Aufgabe! Es ist nahezu unmöglich, die Ärztinnen und Ärzte auf der Basis der von ihnen verrechneten Leistungen einem bestimmten Fachbereich zuzuordnen. Doch auch mit den FMH-Titeln lässt sich das Problem nicht lösen, da sich komplexe Fragen stellen: Was ist mit den Internisten, die über einen zweiten Facharzttitel verfügen? Oder den Pädiatern, die doch Spezialisten sind oder den Psychiatern? Wie definiert man die Gynäkologen, bei denen Frauen für die gesamte medizinische Betreuung ihre jährlichen Kontrollen durchführen lassen?

Auch wenn man die Definition des Begriffs «Grundversorgung» den Kantonen überlassen hätte, hätte erneut die Gefahr bestanden, dass es wie beim Zulassungsstopp zu Ungereimtheiten bei der Anwendung des Gesetzes und damit zu Rechtsunsicherheit gekommen wäre.

Wenn das Parlament nicht in der Lage ist, uns eine bessere Lösung zu unterbreiten, fordern wir es auf, im Minimum die medizinischen Fachgebiete, für die der $\mathrm{Zu}$ lassungsstopp künftig nicht mehr gilt, möglichst breit und klar zu definieren. Da kein Steuerungsinstrument zur Verfügung steht, wie wir es vorgeschlagen haben, ist eine solche Definition für die medizinische Versorgung unseres Landes von grösster Bedeutung.

Man muss den Mut haben, sich auf die klinische Realität statt auf theoretische Definitionen abzustützen: Die Bereiche, in denen ein Mangel besteht, und ebenso jene, die nach Auffassung der Bevölkerung zur Grundversorgung gehören, müssen als solche anerkannt und von den rigiden Einschränkungen befreit werden, die mit dem gegenwärtigen Zulassungsstopp verbunden sind.

Die Ärzteschaft und ihr Dachverband sind enttäuscht, aber nicht entmutigt. Obwohl diese neue Vorlage ganz klar am Ziel vorbeischiesst, sind wir bereit, unseren Beitrag zur Lösung der Probleme zu leisten, die durch die Vorlage entstehen.

Wenn das Parlament tatsächlich den Interessen des Landes dienen will, muss es dringend seine Fähigkeit wiedererlangen, überholte Ideologien und Partikularinteressen einzelner Mitglieder zu überwinden - dies ist unser Wunsch und unsere Hoffnung!

Dr. med. Jacques de Haller, Präsident der FMH 